\title{
DETERMINACIÓN DE BUENAS PRÁCTICAS DE ORDEÑO EN UN GRUPO DE GESTIÓN EMPRESARIAL DE GANADEROS DEL ALTIPLANO CUNDIBOYACENSE
}

\section{DETERMINATION OF GOOD MILKING PRACTICES IN A GROUP OF STOCK FARMER MANAGEMENT DEVICES OF THE CUNDIBOYACENSE HIGH ALTITUDE PLAIN}

\author{
Alfonso Calderón Rangel \\ Gabriel Jiménez Pallares ${ }^{2}$ \\ Freddy García Castro
}

\section{RESUMEN}

Para este estudio, se aplicó una encuesta a veinte ganaderos de un grupo de gestión empresarial de ganaderos del Valle de Ubaté y Chiquinquirá (Colombia), que incluyó variables relacionadas con prácticas de prevención y de control de la mastitis bovina, para la obtención de leche de alta calidad. Para el análisis de todas las respuestas, se utilizó estadística descriptiva. Las prácticas ganaderas presentaron diferentes porcentajes de implementación, donde el $10 \%$ realizó el peluqueado de las ubres, el $75 \%$ de los ganaderos evaluados ordenaron correctamente la secuencia de la rutina del ordeño, el $90 \%$ practicó el despunte y alguna práctica de higienización de la ubre, como el lavado o el presellado y segregaron vacas, en casos de mastitis clínica, en el

Médico Veterinario Zootecnista, M.Sc. Profesor U. de Córdoba, Facultad de Medicina Veterinaria y Zootecnia. Departamento de Ciencias Animales. Instituto de Investigaciones Biológicas del Trópico (IIBT), kilómetro 27 vía Ciénaga de Oro. Correo electrónico: alcaran1@yahoo.com

2 Zootecnista, M.Sc. Investigador CORPOICA. Centro de Investigación Tibaitatá kilómetro 14 vía Mosquera, Colombia. Correspondencia: gabriel.jimenezpallares@gmail.com

${ }^{3}$ Médico Veterinario Zootecnista, M.Sc. Investigador CORPOICA. Centro de Investigación en Salud y Producción Animal CEISA. Bogotá, Colombia. Correspondencia: fgarciacastro@gmail.com
70\%. El sellado de los pezones, se efectuó en el $95 \%$ de los casos, como también una correcta cobertura del sellador; la identificación de vacas tratadas con antibióticos, se efectuó en el $75 \%$ de las fincas. El $90 \%$ de los ganaderos conocen el concepto de inhibidores en la leche y la terapia de la vaca seca fue implementada por el 30\% de los productores. Las buenas prácticas de ordeño son aspectos de manejo que todos los productores deben utilizar rutinariamente, con el fin de generar beneficios para la obtención de leche de mejor calidad y es la forma inicial para competir en mercados externos altamente competitivos.

Palabras clave: Buenas prácticas, rutina de ordeño, mastitis bovina, gestión empresarial.

\section{SUMMARY}

For this study, an inquiry was applied to 20 milk producers, pertaining to an enterprise group of management from the Valleys of Ubaté and Chiquinquirá (Colombia), which included variables related to the prevention practice and the control of bovine mastitis with the aim of obtaining an excellent milk quality. For the analysis of all answers a descriptive statistic was used. The cattle management practices, applied by the cattlemen, presented different percentages regarding the implementation: $10 \%$ accomplished the blunting, $75 \%$ of the evaluated cattleman ordered properly the sequence of the milking routine, $90 \%$ practiced the washings or 
the predipping of the udders, a $70 \%$ segregated cows if a clinic mastitis case was detected; the sealing of the nipples took place in a $95 \%$ of the cases, also a correct cover of the sealant was presented. The identification of the treated cows with antibiotic was achieved in a $75 \%$ of the farms, the $90 \%$ of the cattlemen know the concept of milk inhibitors and the dry cow therapy was implemented by the $30 \%$ of the producers. Good milking practices are relevant aspects which should be known and applied routinely by the producers, since they generate benefits for obtaining an excellent milk quality and it conforms also the initial path to compete with highly competitive foreign markets.

Key words: Good practices, milking route, bovine mastitis, farm managemenent.

\section{INTRODUCCIÓN}

La leche es un alimento de alto valor nutritivo y es esencial en la primera fase de vida de todos los mamíferos. En Colombia hace parte del régimen alimenticio en todos los niveles socioeconómicos de la población. La búsqueda de la calidad de la leche es una preocupación fundamental para los productores, profesionales involucrados en las prácticas veterinarias, industriales, procesadoras y consumidores de lácteos (FEDEGAN, 1999). La calidad de la leche involucra características nutricionales, organolépticas, de inocuidad y microbiológicas; los componentes nutricionales comprenden los porcentajes de proteína, grasa, lactosa, sólidos no grasos, sólidos totales, vitaminas y minerales; los parámetros microbiológicos incluyen el número de unidades formadoras de colonias por mililitro (recuento de mesófilos), presencia de gérmenes patógenos, presencia de inhibidores o residuos de medicamentos y las células somáticas, que son un indicador de mastitis.

Cuando los niveles de mesófilos aumentan, las propiedades nutritivas y organolépticas de la leche se alteran. Los microorganismos son normalmente destruidos por la pasteurización, pero en países donde la presencia de enfermedades zoonóticas, como brucelosis y tuberculosis, constituyen un serio problema para la salud pública (Magarinos, 2000). Además, los industriales buscan que sus proveedores suministren la materia prima con bajos recuentos de mesófilos y de células somáticas para la producción de leches ultrapasteurizadas (UHT) y de productos lácteos de alta calidad, pues no existe ninguna técnica industrial que mejore la mala calidad de la materia prima, la tecnología sólo permite conservar la calidad inicial con que se obtiene en la finca (Jiménez \& Schroeder, 1998; Cotrino E Gaviria, 2006).

La inflamación de la glándula mamaria es conocida como mastitis y se caracteriza por presentar alteraciones del tejido glandular e inducir cambios físicos, químicos y bacteriológicos en la leche (Radostits et al. 2002). Con base en términos usados para describir la epidemiología de los patógenos que causan la infección de la glándula mamaria, ha sido dividida en contagiosa y ambiental. Esta clasificación, se soporta en los diferentes agentes infecciosos implicados en la enfermedad y en las medidas de prevención y control (Blowey $\mathcal{E}$ Edmonson, 1999; Philpot $\&$ Nickerson, 2000). La mastitis es la principal causa de pérdidas económicas en la industria lechera, ya que tanto los productores como los procesadores son afectados por la disminución del volumen de leche producida y en la calidad bacteriológica y composicional de la misma (Philpot $E$ Nickerson, 2000; Calderón et al. 2003).

Entre los factores que han sido considerados importantes en la obtención de leche de excelente calidad está el ambiental que rodea a las vacas, ya que éste las expone a diferentes agentes etiológicos causantes de mastitis. La rutina de ordeño que se definió como la realización de diferentes prácticas de manejo, que incluyen la formación de grupos de ordeño, el despunte, la segregación de casos clínicos de mastitis, la higiene que se logra con el lavado o la implementación del presellado con los pezones y el secado de estos, tiene como objetivo el ordeño de pezones limpios, secos y sanos, con el fin de prevenir y controlar la mastitis y en consecuencia la obtención de leche con bajos recuentos de mesófilos (Erskine et al. 1992).

La implementación de un orden para el ordeño de las vacas, comenzando por las de primer parto, luego con vacas sanas y finalizando con vacas enfermas o con tratamientos con antibióticos Rebhum et al. (1995) y Radostits et al. (2002), demostraron una reducción de los casos de mastitis, debido a que se logra evitar la exposición de las vacas sanas a los microorganismos presentes en las ubres de las infectadas (Kirk et al. 1994). No realizar esta secuencia ayuda a la diseminación de 
infecciones por Staphylococcus aureus, pues se puede propagar hasta la quinta o sexta vaca, siguiente a la infectada, por medio de las pezoneras (Phillips, 1982 citado por Mein, 1998).

El despunte, que consiste en la eliminación de los primeros chorros de leche al iniciar el ordeño, con el fin de disminuir los altos recuentos de bacterias, también permite visualizar cambios físicos en la leche, detectar mastitis clínica y segregar o apartar la(s) vaca(s) para que sea(n) ordeñada(s) al final o en un solo puesto; es una práctica fácil de realizar y de bajo costo (Philpot $E$ Nickerson, 2000).

La higiene y el secado de los pezones es una práctica que reduce y/o elimina la carga bacteriana presente. La higiene de los pezones involucra el lavado o el presellado, donde esta última práctica puede reemplazar al lavado con agua, debido a que se sumergen los pezones dentro de una solución antiséptica, entre 20 y 30 segundos de contacto con la piel luego se retira por medio de una toalla de papel. Esta práctica presenta una mayor efectividad germicida.

Además de los pasos anteriormente mencionados en la preparación de una excelente rutina de ordeño, se debe incluir el flameado o peluqueado de la ubre, que favorece los procesos de higienización de los pezones, debido a que los pelos largos recogen suciedad y dificultan la desinfección, incrementando el riesgo de infección mamaria (Kruze, 1998; Philpot \& Nickerson, 2000); el uso de equipos de ordeño calibrados; la implementación de la terapia de la vaca seca (TVS), que consiste en la aplicación de un antimastítico por vía intramamaria en cada uno de los cuartos al finalizar la lactancia, previa desinfección de los pezones, se introduce parcialmente la cánula, se sellan los pezones y no se vuelve ha ordeñar la vaca sino hasta el próximo parto; tratamiento oportuno de los casos clínicos y, por último, el descarte de vacas con infecciones crónicas (Philpot E Nickerson, 2000).

La aplicación de un producto sellador de pezones debe cubrir la totalidad del pezón, con el proaósito de disminuir al mínimo el riesgo de contagio, debido a que los surcos, las grietas y las heridas de la piel son lugares que se pueden contaminar. Para lograr una buena cobertura del sellador es necesario sumergir la totalidad del pezón dentro de una solución yodada en una copa de no retorno, lo cual reduce el riesgo de infección después del ordeño y sirve para curar las heridas en la piel de los pezones (Kirk et al. 1994; Philpot \& Nickerson, 2000).

La realización de la prueba California para mastitis (CMT), para detectar formas subclínicas de la enfermedad, es otra práctica de prevención y control, la cual presenta grandes ventajas, como el bajo costo; es realizada en cada cuarto de la ubre antes del ordeño y los resultados se obtienen de inmediato, entre otras bondades (Sandholm et al. 1995; Blowey \& Edmonson, 1999).

La implementación de encuestas para obtener información ha tenido un amplio uso en la práctica veterinaria y en la producción animal; por ejemplo, en el área de la salud animal, se han empleado para el desarrollo de estudios de prevalencia y en el campo de la zootecnia han servido para caracterizar los sistemas de producción animal de una región; también se puede realizar conjuntamente en salud y en producción.

Calderón et al. (2003; 2005) determinaron en el altiplano cundiboyacense, por medio de este instrumento y la implementación de un diagnóstico de mastitis, los factores más importantes de riesgo. Así, se observó que las diferentes prácticas que integran el sobreordeño, como ordeño en seco, definido como la visualización del colector sin leche con el equipo ordeñando normalmente, la sobremanipulación de las unidades de ordeño por parte de los operarios, el masaje sobre la ubre, o la combinación de las anteriores, hicieron que las vacas, en primera instancia, presentaran 2,89 veces más probabilidades de enfermar de mastitis en comparación con vacas en las que no se observó ninguna forma de sobreordeño. El inadecuado funcionamiento del regulador, a menos del $90 \%$, debido a una mala ubicación de él o de sus sensores o cuando la capacidad fue menor que la(s) bomba(s) de vacío, ocasionó el aumento en 1,44 veces las probabilidades de presentar mastitis en comparación de las vacas ordeñadas con equipos, en las que los pulsadores funcionaron correctamente. Estos ejemplos hacen que la aplicación de las encuestas en los proyectos de investigación en salud y en producción animal continúen siendo válidos.

La educación continuada por medio de conferencias y de visitas dirigidas a productores y asistentes técnicos es una necesidad, debido a que el fin de estos eventos es la actualización y el reforzamiento de conceptos. La Corporación Colombiana de Investigación Agropecuaria CORPOICA y la Federación Colombiana de Ganaderos 
FEDEGAN, propendieron porque ganaderos y asistentes técnicos del altiplano cundiboyacense (región andina colombiana), se organizaran en un grupo de gestión empresarial (GGE) para que adoptaran elementos de gestión empresarial en sus fincas, con el objetivo de mejorar los procesos en la obtención de leche de excelente calidad y aumentar su competitividad y, especialmente, a personas que administran empresas ganaderas. El objetivo general de este trabajo fue conocer, evaluar y divulgar el grado de adopción de buenas prácticas durante el ordeño.

\section{MATERIALES Y MÉTODOS}

La capacitación impartida a los ganaderos fue realizada mediante la aplicación de módulos de gestión empresarial, de nutrición y de alimentación, salud animal y rutina de ordeño, entre otros. Esta implementación, se realizó por medio de conferencias, de talleres y de visitas a fincas. En la temática de obtención de leche de excelente calidad, al iniciar la capacitación, se implementó una encuesta (Cuadro 1), para conocer las diferentes prácticas de prevención y de control de la mastitis bovina que estaban desarrollando en sus fincas y que los conocimientos adquiridos no influyeran en el diligenciamiento de este instrumento. En las salidas de campo, se observaron y se discutieron las bondades y las dificultades de las diferentes prácticas propuestas durante la realización de este módulo.

Se encuestaron 20 ganaderos y/o asistentes técnicos, de los municipios de Chiquinquirá, Simijaca, San Miguel de Sema, Saboyá, Ubaté, Susa, Cucunubá, Lenguazaque, Fúquene y Guachetá, que pertenecen a las cuencas lecheras de los Valles de Ubaté y Chiquinquirá, región andina colombiana, con alturas comprendidas entre los 2100 y 2700 m.s.n.m.

Las encuestas incluyeron variables cuantitativas y cualitativas; posteriormente, las respuestas fueron tabuladas mediante el uso de una hoja electrónica de Excel y fueron analizadas mediante estadística descriptiva con el uso del programa System Analysis Software (SAS), versión 8.1, USA.

\section{RESULTADOS Y DISCUSIÓN}

En la tabla 1, se presentan los indicadores de manejo general de las fincas y del grupo de gestión empresarial
(GGE), obtenidos durante la realización de los diferentes módulos del proyecto. Por ejemplo, estos productores son generalmente propietarios de los predios, en el $90 \%$ de los casos, en el valle de Chiquinquirá y en el $85 \%$, en el valle de Ubaté. El área promedio de las fincas fue de 21,1 hectáreas para Chiquinquirá y 13,9ha en Ubaté. La topografía predominante de los predios fue plana: en Ubaté fue del $85 \%$ y en Chiquinquirá del $75 \%$.

La producción de leche de las fincas del GGE involucró vacas de la raza Holstein. Mediante pastoreo de praderas de kikuyo (Pennisetum clandestinum), gramínea naturalizada, que se encontró en el $100 \%$ de las fincas; el 55\% de los encuestados respondió que esta especie es explotada como monocultivo en las fincas y el $45 \%$ de los casos estaba asociada con otras especies forrajeras, principalmente raigrases (Lolium sp.) y tréboles blanco y rojo (Trifolium pratense, T. repens).

Los ingresos de las fincas encuestadas, en el $70 \%$ de los casos, se obtienen únicamente por la venta de leche, mientras que el $30 \%$ de los predios los ingresos dependen de la venta de leche y de productos agrícolas. La totalidad de los ganaderos encuestados en el valle de Ubaté venden la leche directamente a la industria láctea, mientras en el valle de Chiquinquirá el $60 \%$ lo hace de forma directa a empresas lácteas y el $40 \%$ a intermediarios.

La evaluación de las diferentes prácticas de prevención y de control de la mastitis bovina en fincas de ganaderos asociados al GGE en los valles de Ubaté y Chiquinquirá, se presentan en la tabla 2.

El flameado o peluqueado de las ubres lo realizaron el $10 \%$ de los participantes del GGE; el $25 \%$ de los ganaderos informaron que sólo realizaron el corte de los pelos de la borla de la cola de las vacas, lo cual se puede explicar por la facilidad para realizar esta labor y por no requerir personal adicional ni equipo especializado. Calderón (2002) determinó que en las fincas donde se implementó el flameado o peluqueado de las ubres, hizo que las vacas presentaran 0,5 veces menos probabilidades de infectarse de mastitis.

Cuando se evaluaron los pasos para realizar una excelente rutina de ordeño, el $75 \%$ de los ganaderos los organizó correctamente. Calderón (2002) encontró que los ganaderos y los asistentes técnicos en las fincas 


\section{Cuadro 1}

ENCUESTA No __ Zona

1- ¿El predio es de su propiedad?

Si O

No $\mathrm{O}$

2- ¿La principal actividad pecuaria es la venta de leche?

Si O

No $\mathrm{O}$

En caso de ser su respuesta ser No,

¿Cuál es la principal actividad?

3- ¿Cuántos litros de leche produce al día?

4- Número de vacas en la finca

5- Número de vacas en ordeño

6- ¿Vende la totalidad de la leche a una sola empresa o persona?

Si O No O

7- ¿Quién es su comprador de leche?

Pasteurizadora $\mathbf{O}$ Intermediarios $\mathbf{O}$ Fabrica manufacturera de quesos $\mathbf{O}$

8- ¿Le bonifican por algún parámetro de calidad?

9- Los pastos predominantes en su finca son Kikuyo $\mathbf{O}$ Ray grass $\mathbf{O}$

Falsa poa $\mathbf{O}$ Azul orchoro $\mathbf{O}$ Festucas $\mathbf{O}$ Trébol blanco Trébol rojo $\mathbf{O}$ Alfalfa $\mathbf{O}$

10- El tipo de ordeño es Manual O Mecánico O

11- ¿Hay una secuencia al comenzar el ordeño de las vacas?

12- Organice los pasos que usted realizar durante el ordeño.

a- sellado b- lavado o presellado c- despunte $d$ - secado

13- ¿Realiza el peluqueado de las ubres?

14- ¿Realiza el flameado de las ubres?

15- ¿Realiza el recorte de los pelos de la borla de la cola?

16- ¿Hace el despunte?

17- ¿Segrega vacas al despunte con cambios visibles en la leche?

18- ¿Lava los pezones antes del ordeño?

19- ¿Hace presellado?

¿Con qué producto?

20- ¿Seca pezones?

21- ¿ildentifica las vacas en producción tratadas con antibiótico?

22- ¿Cómo es la identificación de esas vacas?

23- ¿Considera a los antibióticos como inhibidores de la leche?

24- ¿Cuándo hace tratamientos con antibióticos, entrega esa leche a las empresas que le compran la leche?

En caso de ser su respuesta afirmativa ¿Por qué?

Si O No O

Si O No O

Si O No O

Si O No O

Si O No O

Si O No O

Si O No O

Si O No O

Si O No O

Si O No O

Si O No O

Si O No O

Si O No O

25- ¿Sabe qué es la terapia de la vaca seca?

Si O No O

Si O No O

26- ¿Realiza la terapia de la vaca seca (TVS)?

Si O No O

27- La mejor forma para secar la producción de leche es la TVS

Si O

No $\mathrm{O}$

29- La cobertura del pezón alcanza

Todo el pezón $\mathbf{O}$ La mitad del pezón $\mathbf{O}$ 2/3 del pezón $\mathbf{O}$ La punta del pezón $\mathbf{O}$

30- Las copas del sellador de pezones es?

31- ¿Con qué producto hacen el sellado de los pezones?

32- ¿Lava las copas del sellador de pezones?

Retorno O No retorno O

¿Con qué frecuencia lava estas copas?

33- ¿Tiene tanque frío para almacenamiento de la leche?

Si O No O

Si O No O 
Tabla 1. Características de las fincas de ganaderos asociados al grupo de gestión empresarial en los valles de Ubaté y Chiquinquirá.

\begin{tabular}{|l|c|c|}
\hline \multicolumn{1}{|c|}{ VARIABLE } & CHIQUINQUIRÁ & UBATÉ \\
\hline Es propietario de finca (\%) & 90,0 & 80,0 \\
\hline Ingresos & & \\
\hline \multicolumn{1}{|c|}{ Únicamente por venta de leche (\%) } & 70,0 & 70,0 \\
\hline \multicolumn{1}{|c|}{ Venta de leche y cultivos (\%) } & 30,0 & 30,0 \\
\hline Área de la finca (hectáreas) & 21,1 & 13,9 \\
\hline Topografía plana (\%) & 75,0 & 85,0 \\
\hline Presencia de kikuyo (\%) & 100,0 & 100,0 \\
\hline Monocultivo de kikuyo (\%) & 60,0 & 25,0 \\
\hline Tipo de ordeño: & & \\
\hline & 70,0 & 30,0 \\
\hline Manual(\%) & 10,0 & 90,0 \\
\hline Conocánico(\%) & 95,0 & 100,0 \\
\hline
\end{tabular}

Tabla 2. Resultados de la evaluación de las diferentes prácticas de prevención y control de la mastitis bovina en fincas de ganaderos asociados al grupo de gestión empresarial en los valles de Ubaté y Chiquinquirá.

\begin{tabular}{|c|c|c|}
\hline VARIABLE & $\mathbf{n}$ & $\%$ \\
\hline ¿Realizan peluqueado o flameado de las ubres? & 2 & 10,0 \\
\hline ¿Efectúan el recorte pelos de la borla? & 5 & 25,0 \\
\hline ¿Implementan una secuencia del ordeño correcta? & 15 & 75,0 \\
\hline ¿Hacen el despunte? & 18 & 90,0 \\
\hline ¿Segregan casos clínicos? & 14 & 70,0 \\
\hline ¿Implementan prácticas de higienización de pezones? & 18 & 90,0 \\
\hline ¿Hacen el lavado de los pezones? & 12 & 60,0 \\
\hline ¿Hacen el presellado de los pezones? & 6 & 30,0 \\
\hline ¿Sellan los pezones al finalizar el ordeño? & 19 & 95,0 \\
\hline ¿El sellador es un yodó foro? & 16 & 80,0 \\
\hline ¿El sellador es ácido sulfónico? & 3 & 15,0 \\
\hline ¿Es adecuada la cobertura del sellador de pezones? & 13 & 65,0 \\
\hline ¿Es inadecuada cobertura sellante de pezones? & 7 & 35,0 \\
\hline ¿Realizan el CMT? & 9 & 45,0 \\
\hline ¿Identifican vacas tratadas con antibióticos? & 18 & 90,0 \\
\hline ¿Conocen el concepto de la terapia de la vaca seca? & 18 & 90,0 \\
\hline ¿Implementan la terapia de la vaca seca? & 6 & 30,0 \\
\hline ¿Conocen concepto presencia de inhibidores en leche? & 20 & 100 \\
\hline
\end{tabular}


del altiplano cundiboyacense le dan más importancia a la velocidad que a la calidad con que se hace este trabajo, labor dispendiosa y exigente; añade, además, que cuando los ordeñadores no son estimulados económicamente, en muchos casos, se limitan a realizar una labor repetitiva, sin efectuar una buena limpieza.

En las fincas en la que se realizó este estudio fue una práctica poco común establecer un orden de ordeño para las vacas, pues tan solo el $40 \%$ de las fincas adaptan esta actividad, que puede reducir la prevalencia de las mastitis contagiosas. También se encontró la no implementación de la segregación, ya que los ordeñadores, en su práctica manual, siempre tratan de ordeñar las mismas vacas.

El uso o empleo del despunte, se hizo en el $90 \%$ de las fincas evaluadas, método ampliamente difundido para la eliminación de los primeros chorros de la leche, principalmente. Phelps (1993) afirmó que no realizar el despunte implica pasar por alto un $50 \%$ de los casos clínicos leves de mastitis, porcentaje que indica que los ganaderos son más conscientes de los beneficios que genera esta práctica en la producción de leche de mejor calidad. Esta cifra encontrada en el GGE fue superior a la reportada por Calderón (2002), que tan sólo fue del $67,5 \%$ y a la de Ruegg (2003) para productores del estado de Wisconsyn, quienes participaron en programas de extensión e indicaron que el $31 \%$ no implementaron el despunte, con el fin de ganar tiempo en el ordeño y hacer una mayor ocupación de las salas de ordeño. Calderón (2002), en el altiplano cundiboyacense, reportó que la realización del despunte hizo que las vacas presentaran 0,54 menos probabilidades de infectarse o presentar mastitis.

Durante el ordeño, la segregación de las vacas infectadas con mastitis clínica fue realizada en el $70 \%$ de las fincas de los ganaderos encuestados del GGE. Es posible que algunos ordeñadores descarten los primeros chorros de leche, obviando su evaluación, por lo tanto, es posible que ordeñen vacas con mastitis clínica. Kirk et al. (1994) observaron que la segregación puede hacer disminuir en 0,81 veces las probabilidades de presentar mastitis. Lo recomendando es destinar unidades para el ordeño de vacas enfermas o crear lotes de ordeño, lavar las unidades entre vacas o ensamblar un sistema de lavado mecánico, para evitar el traspaso de infecciones, por medio de las pezoneras contaminadas (Phillips 1982, citado por Mein, 1998). Cassel et al. (1994) aconsejan, para ordeños con un gran número de vacas, crear grupos separados de vacas con y sin mastitis, como alternativa para disminuir la incidencia por microorganismos contagiosos.

El lavado y el secado de las ubres, se efectuó en el $60 \%$ de las fincas de los productores del GGE; el 30\% realizó el presellado y en el $10 \%$ no se hizo ninguna práctica de higienización. Calderón (2002) estableció que en fincas donde esta práctica se implementó, las vacas disminuyó en 0,72 veces la probabilidad de presentar mastitis. Burmeister et al. (1998) encontraron que la acción del presellador hizo que las vacas presentaran 0,54 veces menos probabilidad de infectarse de mastitis. Gélves E Pérez (1998) hallaron que la no implementación de esta práctica aumentó en 2,2 veces la probabilidad de infección en el momento del ordeño.

En el valle de Ubaté, se determinó que el $90 \%$ de los ganaderos participantes del GGE, el ordeño se realizó con equipo mecánico, mientras en el valle de Chiquinquirá, en el $70 \%$ de las fincas, predominó el ordeño manual. CORPOICA (1995) encontró que la Sabana de Bogotá es la zona del país que presenta la mayor mecanización del ordeño, seguida del valle de Ubaté y de Chiquinquirá. El promedio de hembras en ordeño en estos dos valles, para este grupo de ganaderos fue de 28 vacas y la producción diaria de leche osciló entre 10 y 18 L/vaca-día, rango que está por encima del reporte de CORPOICA (1995) de 9,7L/vaca-día y dentro de los reportes de Gomezjurado $\mathcal{E}$ Castillo (1997) y Calderón (2002) para vacas Holstein, cuya fuente principal de forrajes en el altiplano cundiboyacense es el pasto kikuyo.

Finalizado el ordeño, en sistemas especializadas, se debe implementar el sellado de los pezones, el cual se efectuó en el 90\% de las fincas del GGE. Calderón (2002) estableció que donde esta práctica se llevó a cabo las vacas disminuyeron en 0,65 veces la probabilidad de padecer mastitis.

Una adecuada cobertura del sellador, se presentó en el $65 \%$ de los productores evaluados, mientras el $30 \%$ cubrió el primer tercio distal del pezón, lo que se consideró como inadecuado.

Al preguntar a este grupo de ganaderos por la realización de la prueba California para mastitis (CMT), el $45 \%$ respondió que implementan esta prueba de diagnóstico 
y la región geográfica que presentó la mayor frecuencia fue el valle de Ubaté. Este menor porcentaje hallado respecto a lo reportado por Calderón (2002), que fue del $90 \%$, se debió a que los ganaderos del GGE no estaban siendo motivados económicamente a producir leche de mejor calidad. Zapata E Schachtebeck (1996) determinaron, para esta misma región, que el $64 \%$ de los ganaderos visitados por ellos, no realizaron esta práctica, por carecer de personal capacitado y también por la falta de motivación económica en la región.

Una de las formas de prevenir la presencia de inhibidores en la leche es la identificación de las vacas tratadas con antibióticos. Cuando se preguntó al respecto, el $90 \%$ de los ganaderos contestaron que realizan esta labor; la forma de identificación, se basó en registros y con algún tipo de marca (cinta, bandas), mientras que en el $10 \%$ de los productores confió en la memoria de los operarios. Es ideal que las marcas que se hagan en las vacas tratadas con antibióticos sean visibles fácilmente y que perduren, con el fin de que cualquier persona que no esté familiarizado con el ordeño pueda segregar a las vacas tratadas y descartar su leche.

El 90\% de los ganaderos del GGE respondieron que la mejor alternativa para secar la producción de leche de una lactancia es la terapia de la vaca seca; Calderón (2002) determinó que la implementación del secado drástico hizo que presentaran 0,84 veces menos probabilidades de enfermar por mastitis y la aplicación de la TVS disminuyó en 0,51 veces probabilidades de presentar la enfermedad. El 30\% de los ganaderos tienen alta confianza en la realización de esta práctica y la aplican a la totalidad de las vacas que finalizan un ciclo productivo; el $10 \%$ de los ganaderos no la conocían.

Los ganaderos del GGE conocen el concepto de inhibidores; cuando la leche es comprada por empresas que pagan por calidad y realizan análisis para su determinación descartan esta leche; sin embargo, la venden con inhibidores, cuando los agentes comercializadores no realizan los respectivos controles, es decir no descartan esta leche.

\section{CONCLUSIONES}

Se observó que los ganaderos son cada día más conscientes de las bondades y los beneficios que generan las buenas prácticas ganaderas en la obtención de leche de mejor calidad y que la educación continuada tiene alta prioridad en formación y en capacitación del recurso humano que labora en las fincas.

Es indispensable implementar una excelente rutina de ordeño en todas las empresas ganaderas, dedicadas a la producción de leche; para lo cual, se deben definir, previamente, las acciones que se van a efectuar en el proceso del ordeño, punto de partida para la producción de leche, con altos estándares de calidad.

Los ganaderos del GGE han implementado buenas prácticas en sus fincas, con el propósito de producir leche de buena calidad, a pesar de que muchos de ellos no reciben bonificaciones por parte de agentes compradores de leche.

Una estrategia visualizada fue la asociación de pequeños y medianos productores, para que éstos puedan ofrecer un mayor volumen y mejor calidad de la leche entregada, esto les da poder de negociación frente a la industria láctea, y así se les reconozca el esfuerzo diario en la producción de leche de buena calidad.

En el marco de la producción de alimentos inocuos, el concepto de inhibidores se debe ampliar, además del uso y el manejo de antibióticos a otros productos químicos, como los antiparasitarios, de uso interno y externo, los detergentes y desinfectantes, empleados en la limpieza y la desinfección de diferentes utensilios y la estimulación económicamente a los ganaderos para descartar estas leches para consumo humano, así como también transferir las implicaciones de los inhibidores en la salud humana.

\section{BIBLIOGRAFÍA}

BLOWEY, R.; EDMONSON, P. 1999. Control de la mastitis en granjas de vacunos de leche. Guía práctica e ilustrada. Editorial Acribia, S.A. Zaragoza, España. 208p.

BURMEISTER, J.E.; FOX, L.K.; HILLERS, J.K.; HANCOCK, D.D. 1998. Effects of premilking and posmilking teat desinfectans of teat skin condition. J. Dairy Sci. 81(7):1010-1916.

CALDERÓN, R.A. 2002. Cuantificación de factores de riesgo de mastitis en sistemas de producción élites en el altiplano Cundiboyacense. Tesis de Maestría. 
Facultad de Medicina Veterinaria y de Zootecnia, Universidad Nacional de Colombia. 232p.

CALDERÓN, A.; DONADO, P.; GARCÍA, G.; GARCÍA, F. 2005. Modelo de regresión logística para determinar relaciones de equipos de ordeño con lapresentación de la mastitis bovina en fincas del altiplano cundiboyacense. Rev. Col. Cienc. Pec. 18(1):11-16.

CALDERÓN, A.; DONADO, P.; JIMENES, G.; GARCÍA, G.; GARCÍA, F. 2003. Evaluación de los equipos de ordeño y sus implicaciones en la presentación de la mastitis bovina en el altiplano cundiboyacense. Rev. Med. Vet. Zoot. Univ. Nac. 50(2):35-42.

CASSEL, E.K.; VOUGH, L.R.; VALNER, M.A.; EICKELBERGER, R., PETER, R.R. 1994. A demonstration project of interdisciplinary dairy herd extension. Advising finded by industry and user. 3. impact on management practices. J. Dairy Sci. 77:24612476.

CORPORACIÓN COLOMBIANA DE INVESTIGACIÓN AGROPECUARIA (CORPOICA). 1995. Entorno socioeconómico y problemática tecnológica de la ganadería de leche especializada. Programa regional pecuario. Regional Uno, Corpoica, Tibaitatá. 94p.

COTRINO. V.; GAVIRIA, C. 2006. La calidad de la leche cruda y el tratado de libre comercio. Disponible desde Internet en http://mvltda. com/programas /ar05.html\#top (con acceso el 15/01/07)

ERSKINE, R.J.; WILSON, R.C.; RIDDELL, R.G.; TYLER, J.W.; ASPEARS, H.J.; DAVIS, B.S. 1992. Administration of gentamicin as treatment for experimentally induced Escherichia coli mastitis in cows. Am J. Vet. Res. 53(3):375-381.

FEDERACIÓN COLOMBIANA DE GANADEROS (FEDEGAN). 1999. La ganadería bovina en Colombia 1998-1999. Bogotá, Colombia. 30p.

GÉLVES, H.J.E.; PÉREZ, E.P.B. 1998. Ensayo clínico para la evaluación de diferentes preparados antimicrobianos como tratamiento de la vaca seca en hatos lecheros en la Sabana de Bogotá. Tesis de Grado. Fac. Med. Vet. Y Zootecnica. U.N. de Colombia. 223p.

GOMEZJURADO, H.J.; CASTILLO, T.S.T. 1997. Análisis técnico económico de dos modelos representativos del sistema de producción de leche en la Sabana de Bogotá. Programa Regional de Sistemas de producción. Regional Uno, Corpoica, Tibaitatá. 58p.

JIMÉNEZ, G.; SCHROEDER, B. 1998. La calidad de la leche: base de la competitividad. Revista Costa Ganadera (Barranquilla, Colombia). 10(36):10$11 ; 37-38$.

KIRK, J.H.; DEGRAVES, F.; YLER, J. 1994. Recent progress in treatment and control of mastitis in cattle. J. Am. Vet. Med. Assoc. 204(8):1152-1158.

KRUZE, J. 1998. La rutina de ordeño y su rol en los programas de control de mastitis bovina. Arch. Med. Vet. 30(2):07-16.

MAGARINOS, H. 2000. Producción higiénica de la leche cruda. Una guía para la pequeña y mediana empresa. Ed. Producción y Servicio Incorporados, S.A. Guatemala.

MEIN, G. A. 1998. Nuevos estándares y pautas para los sistemas de ordeño. En: $1^{\text {er }}$ Seminario internacional. Gerenciamiento de operaciones para obtener leche bajo los parámetros de calidad total. Fisiología y fisiopatología de la lactancia y el ordeño. Pergamino, Argentina. p.191-196.

PHELPS, A. 1993. Mastitis. Agricultura de las Américas. Jul/Ago: 77:82.

PHILPOT, N.; NICKERSON, S. 2000. Mastitis: Ganado la lucha contra la mastitis. Babson Bros. Co. USA. $192 p$.

REBHUM, W.C.; GUARD, C.; RICHARDS, C.M. 1995. Disease of dairy cattle. Lea and Febiger, USA. p.253-308.

RADOSTITS, O.M.; GAY, C.C.; BLOOD, C.D.; HINCNCLIFF, K.W. 2002. Medicina Veterinaria 9ª Edición. 
Ed. McGraw-Hill-Interamericana S.A. Madrid, España. p.711-779.

RUEGG, P.L. 2003. El Papel de la higiene en el ordeño eficiente. Ordeño y calidad de leche No. 406. Instituto Babcock, Universidad de Wisconsyn.

SANDHOLM, M.; HONKANEN-BUSALSKI, T.; KAARTINEN, L.; PYÖRÄLÄ, S. 1995. The bovine udder and mastitis (Finland). University of Helsinki, Faculty of Veterinary Medicine. 312p.
ZAPATA, O.H.; SCHACHTEBECK, R.E. 1997. Estudio sobre la prevalencia de seropositividad a Brucella abortus y Leptospira interrogans en 11 ganaderías selectas en los valles de Ubaté y Chiquinquirá. Tesis de grado. Facultad de Medicina Veterinaria y de Zootecnia, Universidad Nacional de Colombia. p.107-108.

Recibido: Septiembre 17 de 2007

Aceptado: Abril 7 de 2008 